OPEN ACCESS

Edited by:

Ghanshyam Upadhyay,

City University of New York, USA

Reviewed by:

Ravi C. Kalathur,

New York Structural Biology Center,

USA

*Correspondence:

Arun Chaudhury

arunchaudhury.boston@gmail.com

Specialty section:

This article was submitted to

Gastrointestinal Sciences,

a section of the journal

Frontiers in Physiology

Received: 26 July 2016 Accepted: 13 October 2016

Published: 28 October 2016

Citation:

Chaudhury A, Dendi VSR and Mirza W (2016) Colligative Property of ATP:

Implications for Enteric Purinergic

Neuromuscular Neurotransmission

Front. Physiol. 7:500

doi: 10.3389/fphys.2016.00500

\section{Colligative Property of ATP: Implications for Enteric Purinergic Neuromuscular Neurotransmission}

\author{
Arun Chaudhury ${ }^{1 *}$, Vijaya S. R. Dendi ${ }^{2}$ and Wasique Mirza ${ }^{3}$ \\ ${ }^{1}$ GIM Foundation, Little Rock, AR, USA, ${ }^{2}$ Christus Trinity Mother Frances Hospital, Tyler, TX, USA, ${ }^{3}$ The Wright Center for \\ Graduate Medical Education, The Commonwealth Medical College, Scranton, PA, USA
}

Keywords: SLC17A9, VNUT, neurotransmission, transporter, enteric nervous system

\section{ATP, A COMMON CONSTITUENT OF VESICLES, REDUCES INTRAVESICULAR OSMOTIC PRESSURE BY POLYMERIZING VESICULAR CONTENTS AND REDUCING THE NUMBER OF INDIVIDUAL FREE PARTICLES}

A recent report by Estévez-Herrera et al. (2016) suggested that ATP, the energy coin, also a neurotransmitter, controls the osmotic pressure of vesicular contents (Estévez-Herrera et al., 2016). Since the discovery and description of quantal transmission (Katz, 1971; Bennett and Kearns, 2000), membrane-delimited packeted structures, the vesicles, have been morphologically identified and correlated with secretion in several secretory tissues, including nerve terminals of central and peripheral neurons, chromaffin cells, platelets, and insulin-secreting beta cells of the pancreas (Goyal and Chaudhury, 2013; Südhof, 2013; Thorn et al., 2016). Though it is now known for some time that ATP is concentrated in the vesicles, the demonstration that ATP, by virtue of being negatively charged, and its ability to associate with positively charged molecules like amines, agglomerates vesicular particles, is a significant finding. Vesicles do not release their contents randomly within the cytosol, but rather are transported to the membranes for exocytosis (Jena, 2009). An important contributor to this site-specific release of vesicular contents may be the constant fine tuning and maintenance of osmotic pressure isotonic with the cytosol of the vesicle-containing structure. In the light of such plausible dynamic regulation of osmotic pressure of vesicles, the demonstration of the likely role of ATP in regulating this vesicular osmotic pressure acquires importance (Estévez-Herrera et al., 2016). In this perspective, we discuss the implications of these findings on enteric purinergic inhibitory musculomotor neurotransmission.

\section{VESICLE MEMBRANE INTEGRITY IS MAINTAINED BY LOWERING INTRAVESICULAR OSMOTIC PRESSURE}

Osmotic property is a colligative property: it depends on the number of particles, as suggested by Raoult:

$\mathrm{pV}=\mathrm{nRT} . .$. standard gas equation

$\pi \mathrm{V}=\mathrm{nRT} \ldots . . . \pi=$ osmotic pressure

$\pi=[\mathrm{n} / \mathrm{V}] \mathrm{RT}$...note the dependence of $\pi$ on $\mathrm{n}$, the number of particles, the essence of colligative property

The process of release of neurotransmitters is highly coordinated, involving several 100 proteins with graded responses to intracellular calcium fluctuations (Goyal and Chaudhury, 2013; Südhof, 2013). In neurosecretory processes like stimulation-evoked neurotransmission, the pool of readily 
releasable vesicles empty contents after docking at the cell membrane of the active zones. Electron micrographs of nerve terminal varicosities always demonstrate intact membranes of vesicles within the cytosol of the terminal (Collman et al., 2015). This structural integrity of vesicles strongly suggests that the contents of the vesicles are isotonic with the matrix of the varicosities.

Earlier, an interesting study investigated the osmotic pressure of synaptic vesicles (Kopell and Westhead, 1982). This study revealed that the vesicles obtained from chromaffin cells of the adrenal gland, despite their high concentrations of various amines, and peptides, remained isotonic (Kopell and Westhead, 1982). This study hypothesized that the highly negatively charged ATP molecules, a major co-constituent of chromaffin cells beside the positively-charged amines, forms a polymeric complex within the vesicles (Kopell and Westhead, 1982).

The leading hypothesis by Estévez-Herrera et al. (2016) is that when ATP agglomerates the vesicular contents, there is a reduction in the number of free particles, leading to balance of pressures across the vesicular membrane. This physical property may be potentially a critical determinant of membrane integrity of vesicles from their biogenesis until the time they receive the necessary stimuli for exocytosis.

\section{SLC17A9 TRANSPORTS ATP INTO VESICLES}

Estévez-Herrera et al. (2016) show that SLC17A9, the vesicular nucleotide transporter (VNUT; Sawada et al., 2008), performs a rate-limiting step to the transport of ATP within the large dense core (LDC) particles of the chromaffin cells. It has been specifically demonstrated earlier and well-known for some time that the drive for ATP entry is regulated by a proton motive force (Sawada et al., 2008). Thus, it is imperative that the colligative property has a direct relationship with the intravesicular acidity.

\section{ENTERIC INHIBITORY SMOOTH MUSCLE NEUROTRANSMISSION INVOLVES RELEASE OF VESICULAR ATP AND DE NOVO SYNTHESIZED NITRIC OXIDE (NO)}

The biophysical characteristics of particle-based actions of ATP may have important implications for enteric neuromuscular transmission. Evoked enteric inhibitory neuromuscular neurotransmission involves the sequential release of purines (most importantly, ATP) and the gas nitric oxide (NO), synthesized by neuronal nitric oxide synthase (nNOS) at the membranes of nerve terminals (Chaudhury et al., 2011, 2012; Chaudhury, 2014, 2015a, 2016a,b). While ATP is stored in the vesicles of the nerve terminals, NO is synthesized de novo (Chaudhury, 2016a). The released ATP during evoked neurotransmission hyperpolarizes the smooth muscle membrane (Chaudhury, 2016a). In a span of a few $100 \mathrm{~ms}$, the membrane potential endeavors to swing back to its resting stage. However, the prolonged release of nitric oxide prevents the restoration of membrane potential to baseline and aims to maintain the hyperpolarization. This is manifested as the slow inhibitory junction potential (sIJP), unambiguously recorded by several investigators across decades (Figure 1; Bennett et al., 1966; Atanasova et al., 1972; Smith et al., 1990; Hirst et al., 2004; Allego et al., 2008; Chaudhury et al., 2011, 2012; Chaudhury, 2016a). Following the paradigm-shifting demonstration of ATP as a neurotransmitter using gut tissues (Burnstock et al., 1970), there was a gap of several decades in which the VNUT could not be identified within the synaptic vesicles. Quinacrine, the antimalarial drug, robustly stains ATP containing nerve terminals (Belai and Burnstock, 1994), but this never could provide insights into how ATP, a highly negatively charged molecule, could be shuttled across the cell membrane of the vesicles. Following the report by Sawada et al. (2008) of the molecular identity of VNUT as the solute carrier protein SLC17A9 (Sawada et al., 2008), a commercially available antibody was used to demonstrate the existence of SLC17A9 in the enteric musculomotor nerve terminals (Chaudhury et al., 2012), providing the preliminary critical evidence of fulfillment of the Sherringtonian criterion (Levine, 2007) for the existence of the transporter of a neurotransmitter.

\section{IMPORTANCE OF VESICULAR CONTENT CLUSTERING BY ATP IN DIVERSE ENTERIC SYNAPTOSOMAL VESICLES}

ATP is widely distributed in enteric musculomotor nerve terminals. It is present in both VIP containing large dense core vesicles, as well as acetylcholine (Ach) containing small clear vesicles (Figure 1). While VIP plays a significant role in inhibitory neurotransmission and smooth muscle relaxation, Ach facilitates excitatory neurotransmission and smooth muscle contraction. Alternate relaxation and contractions of smooth muscles at the same location are the key factors that determine transit of luminal contents but very little is known regarding the release kinetics of VIP and Ach, and parallel release of ATP. Below, we discuss some of the possibilities that may happen to execute these complex release of excitatory and inhibitory neurotransmitters during enteric nerve-smooth muscle neurotransmission. We also discuss the potential role of colligative property of ATP in influencing these functions.

i VIP containing large dense core vesicles: In the enteric nerve terminals, what potential colligative role does ATP play? The enteric inhibitory neurotransmission is represented electrophysiologically by the fast and slow IJP, mediated by the purine nucleotide ATP and NO, respectively (Chaudhury et al., 2011, 2012; Chaudhury, 2016a). While ATP is released from vesicles (Chaudhury et al., 2012), NO is synthesized by nNOS at the nerve terminal membrane (Chaudhury et al., 2009, 2011; Chaudhury, 2014). It is only scantily known whether other chemicals are co-released with ATP. Classical studies by Whittaker using enteric synaptosomes has demonstrated that many neuropeptides coexist with ATP. One of them is vasoactive intestinal polypeptide (VIP; Agoston et al., 1988; Whittaker, 1989). It is possible that peptide VIP is coreleased with ATP during evoked neurotransmission (Agoston and 

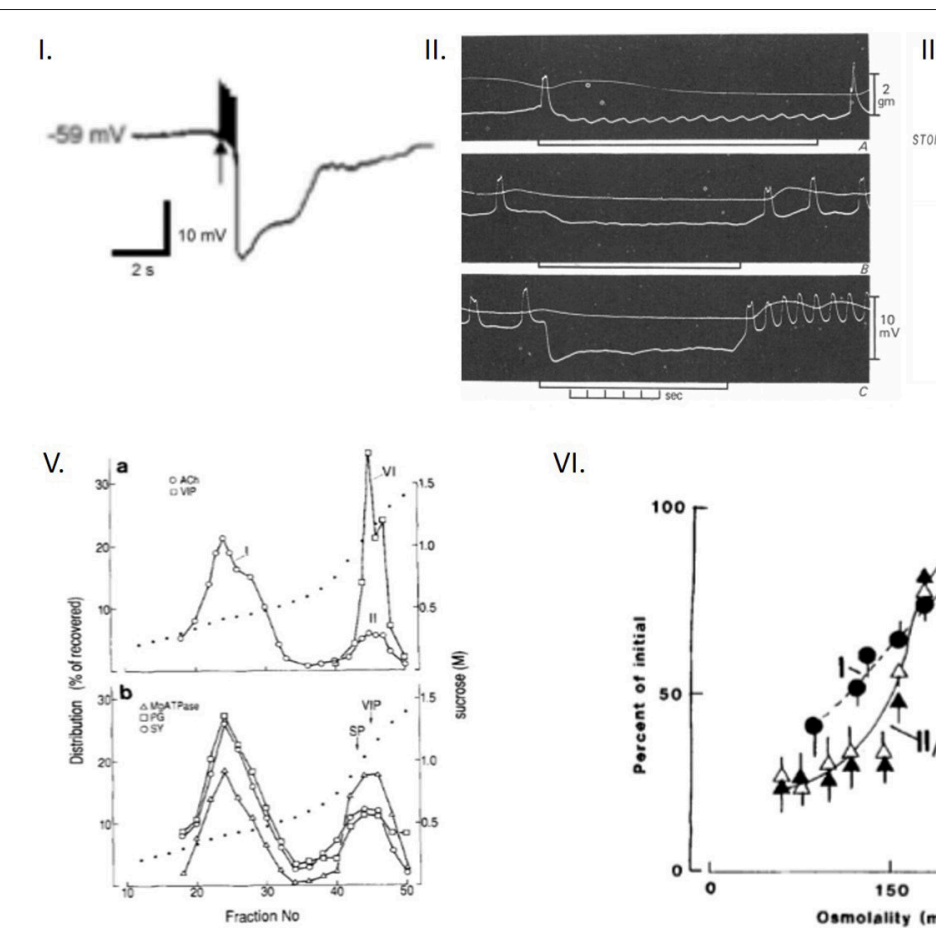

VI.

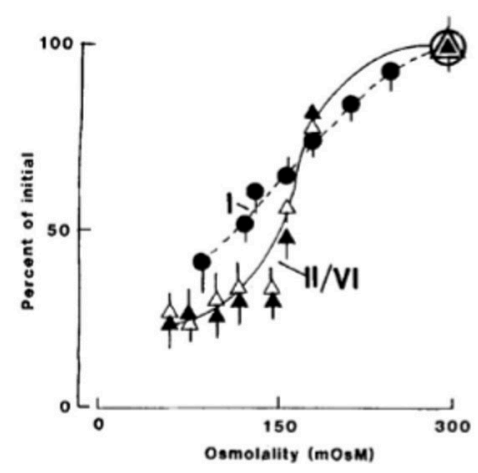

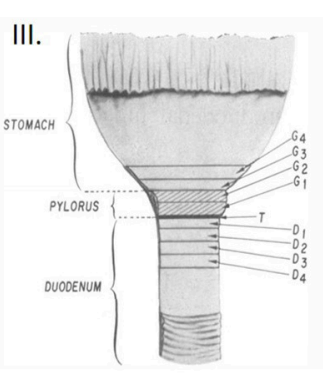

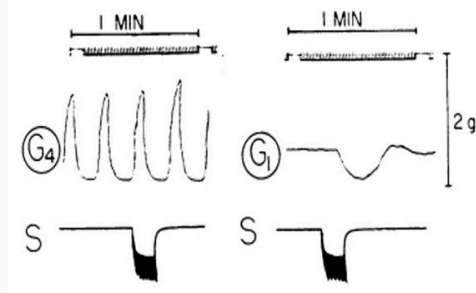

IV.

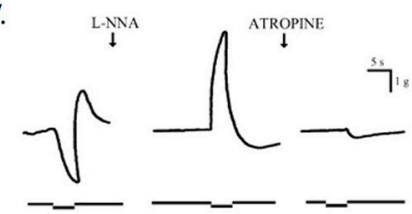

VII.

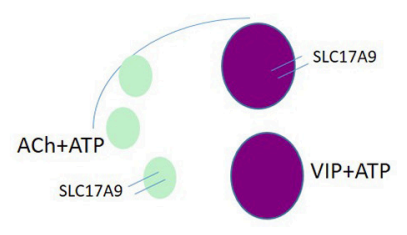

FIGURE 1 | Colligative property of ATP may have important implications for enteric inhibitory neuromuscular neurotransmission. (I) Trace of a compound inhibitory junction potential Note the fast phase of the hyperpolarization (fast IJP, ATP mediated), followed by the slow delayed phase to repolarization (slow IJP, NO mediated). ATP is released from vesicles, whereas NO is synthesized de novo by nNOS. However, the identity of the ATP containing vesicles is not discretely described for myenteric axons and nerve terminals. (II) Three traces of electrical recordings showing differential responses to electrical field stimulation (EFS) intensity The upper trace is the mechanical recording, whereas the lower trace depicts the electrical activity. The three traces corresponds to 1,10 , and $30 \mathrm{~Hz}$ of stimuli, respectively. Note that at the beginning of the stimulus, inhibitory neurotransmission is observed, with hyperpolarization of the membrane potential (inhibitory junction potential, IJP). The tendency to recover to the baseline membrane potential is less with higher intensities of stimuli. The rapid phase of IJP is due to ATP. The slow phase is due to sustained synthesis of NO. However, the identity of the vesicles that releases ATP is not known. A notable feature of this recording is the excitatory junction potential (EJP) at the end of the IJP. EJPs are mainly mediated by acetylcholine. It is possible that Ach is released with the decay of the stimulus. It is also possible that Ach is released initially, but the overwhelming amount of ATP, through its postjunctional effects on the P2Y1 receptor, mediates an inhibitory response. Evidence also exists that the sustained phase of the IJP may be due to a prejunctional modulation by VIP, which is also coreleased with ATP. (III) Further examples of sequential relaxation and contraction during mechanical recordings G1 represents a pyloric strip, whereas G4 represents an antral strip. Note the spontaneous contractions of the antrum. In contrast, the EFS induces relaxation of the pyloric strip, which likely contributes to pyloric patency and gastric emptying in the organ in vivo. (IV) Mechanical relaxations are sensitive to L-NNA, and contractions to atropine Mechanical recordings from lower esophageal sphincter. Again, note the sequential off-contraction following an on-relaxation during the EFS (left panel). The middle panel shows an on-contraction. Combined L-NNA-atropine still manifests residual relaxation. (V) Enteric synaptosomal preparations show distinct vesicular compositions of acetylcholine and VIP Note that the fraction I is composed of only Ach, whereas the fraction II is composed of both Ach and VIP. The significance of this complex composition is not clear, but may potentially contribute to the excitation seen at the tail phase of an IJP. Also note that both fractions associate with $\mathrm{Mg}^{2+}$-ATPase, which is myosin. This could be both myosin Va and myosin II. (VI) Osmotic fragility of enteric synaptosomal vesicles Note that the Ach-VIP containing vesicles are slightly more fragile (as tested by incubation in a hypotonic solution) in comparison to only acetylcholine-containing vesicles, probably due to their large size. Per the recent study of Estévez-Herrera et al. (2016), ATP may importantly contribute to the osmotic stability of these vesicles. (VII) Cartoon depicting the potential contribution of colligative property of ATP to enteric neurotransmission This is a simplified version of what may actually exist in the enteric synaptosomes. The arc represents the active zone of the junctional membrane of the enteric varicosities. Pure ATP containing vesicles have never been detected in myenteric preparations. They either coexist with Ach, VIP or both Ach and VIP (this third kind not shown in the cartoon). ATP, via its colligative property, may contribute to the regulation of release kinetics of either Ach or VIP or both, depending upon the stimulus intensity. Reproduced with permission from Chaudhury et al. (2011); Agoston and Whittaker (1989); Anuras et al. (1974); González et al. (2004); Burnstock (1981).

TABLE 1 | Table showing the relative specific gravity of different enteric synaptic vesicles.

\begin{tabular}{lcccc}
\hline Enteric Neurotransmitter & Ach (acetylcholine) & Substance $\mathbf{P}$ & Somatostatin & VIP (vasoactive intestinal polypeptide) \\
\hline Mean density $(\mathrm{g} / \mathrm{ml})$ & 1.066 & 1.123 & 1.138 & 1.148 \\
Vesicle diameter $(\mathrm{nm})$ & 61 & 65 & 37 & 110
\end{tabular}

The recent study by Estévez-Herrera et al. (2016) suggest that ATP may contribute to the osmotic stability of these vesicles. Data obtained from Agoston et al. (1985). 
Whittaker, 1989). Though VIP may not have a direct impact on the slow IJP, studies have shown the important role of VIP in modulation of presynaptic calcium concentrations, thus having an effect on both exocytosis of ATP, as well as de novo synthesis of NO (Van Geldre and Lefebvre, 2004). This may be a reason for earlier erroneous suggestions of VIP as the enteric inhibitory neurotransmitter (Goyal et al., 1980; Mackenzie and Burnstock, 1980). It is possible that ATP may importantly contribute to the osmotic pressure of the VIP containing large dense core vesicles, which are similar to the chromaffin granules. This remains to be tested.

ii Acetylcholine (Ach) containing small clear vesicles (SCV): An important aspect of coexistence of ATP in the enteric nerve terminals is that with acetylcholine (Ach). ATP, being negatively charged, can associate with the positively charged quaternary ammonium of acetylcholine. Ach contributes to excitatory junction potentials (EJPs) and contractile motor responses (Anuras et al., 1974). Intriguingly, cholinergic vesicles coexist with VIP containing large dense core vesicles (Agoston et al., 1988). The significance of this important observation is unknown. The dynamics of release of ATP/NO and Ach is also not known (Chaudhury, 2016a). A common observation is the occurrence of a contractile response at the end of an episode of relaxation during post-stimulus mechanical recordings of gastrointestinal muscle strips (Figure 1; Anuras et al., 1974). Per the previous observation, it shall imply that a given intensity of stimulus first supports inhibitory neurotransmission, followed by the cholinergic excitatory response. By the time the excitatory response appears, the initial stimulus would start decaying temporally. But what prevents simultaneous release of Ach during ATP release? It has been shown that low frequency electrical field stimulation of synaptosomes ex vivo released ACh by $<$ four-fold the basal release; the simultaneously detected VIP secretion was only slightly raised above the basal level. During high frequency stimulation $(50 \mathrm{~Hz})$, VIP secretion was greatly increased (to five-fold the resting release) whereas the release of ACh increased to only $150 \%$ of the basal output (Agoston and Whittaker, 1989). An alternate possibility is that both ATP and Ach are coreleased, and depending on the postjunctional responses, there is an inhibitory or excitatory response. Sometimes, say during segmentation contractions, a long stretch may simultaneously have sustained inhibitory purinergic-nitrergic responses (Gwynne and Bornstein, 2007). How is cholinergic responses excluded during this activity? In the light of these perspectives, the reductionist concepts of descending inhibitory neurotransmission and ascending excitatory neurotransmission merits critical revision. How do the circuits toggle between an excitatory vs. inhibitory prejunctional release? This may also result from a summative response. Though the current concepts limit us to thinking that the postjunctional smooth muscle responses are somewhat chaotic and stochastic in nature, there is potential stoichiometry to how nature must have designed these enteric circuits, including specific responses to intraluminal stimuli, and responses mediated by intrinsic primary afferent neurons (IPANs). SLC17A9 colocalizes with vesicular acetylcholine transporter (Chaudhury et al., 2012). Again, it remains to be examined whether ATP contributes to colligative actions with the acetylcholine containing vesicles.

\section{IMPACT OF COLLIGATIVE PROPERTY OF ATP ON DIFFERENTIAL RELEASE OF ENTERIC EXCITATORY AND INHIBITORY NEUROTRANSMITTERS AND NEUROMODULATORS}

The biophysical experiments of estimating osmotic pressure of vesicles are challenging to perform, and more so in an in vivo context. Enteric synaptosomal preparations may be used to examine whether the mechanisms of ATP contributing to particle stability (Estévez-Herrera et al., 2016) is a general phenomenon seen across all vesicular structures, for example cholinergic containing small synaptic vesicles and VIP containing large dense core vesicles. Additionally, polymeric vesicular contents with (ATP-neurotransmitter) n needs demonstration, likely by estimation of the polymeric masses or by surrogate measures of vesicular acidity. The specific gravity of the clear and dense core vesicles have been reported (Table 1). A relevant hypothesis that may be examined is whether the ATP contents are different between exclusive Ach containing vesicles vs. AchVIP containing vesicles. If so, the particle aggregating effects of ATP may differentially regulate release of Ach and VIP during excitatory and inhibitory neurotransmission, respectively.

\section{PATHOPHYSIOLOGICAL IMPLICATIONS OF ATP COLLIGATIVE PROPERTY FOR FUNCTIONAL BOWEL DISORDERS: LESSONS MAY BE LEARNT FROM SLC17A9-/- MICE}

Most esophagogastrointestinal motility disorders involve dysfunction of nitrergic biosynthesis and postjunctional smooth muscle responses (Chaudhury, 2015a,b, 2016b). Varied mechanisms of pathophysiology finally converge on the nitrergic pathways to cause diseases like achalasia, gastroparesis, pseudoobstruction, megacolon, and constipation. There are virtually no disorders in which purinergic inhibitory neurotransmission has been found as the solitary basis of the gastrointestinal motility disorder. There are incipient suggestions that the purinergic fast IJP may be impaired, for example in the transitional zone in Hirschsprung's disease (Jiménez et al., 2015). It is possible that defective ATP production or vesicular shuttle may cause subtle defects in inhibitory neuro-smooth muscle neurotransmission. Mitochondrial ATP production is defective in diabetes (Bagkos et al., 2014). ATP gates SLC17A9 (Sawada et al., 2008). Thus, deficient ATP production may cause SLC17A9 channelopathy. SLC17A9 knockout mice do not show any frank gastrointestinal phenotypic abnormalities and have normal body weight (Dr. Richard Palmiter, personal communication). In SLC17A9 knockout mice, insulin vesicular exocytosis is accelerated (Sakamoto et al., 2014). This may result from deficient particle 
aggregating action of ATP. Whether such defects also occur in enteric vesicular release remains to be tested. We plan to undertake further studies of the enteric synaptosomal properties during neurotransmission to test the generalizability of colligative property of ATP and any effect of its deficiency on purinergic neuromuscular transmission.

\section{REFERENCES}

Agoston, D. V., Ballmann, M., Conlon, J. M., Dowe, G. H., and Whittaker, V. P. (1985). Isolation of neuropeptide-containing vesicles from the guinea pig ileum. J. Neurochem. 45, 398-406.

Agoston, D. V., Conlon, J. M., and Whittaker, V. P. (1988). Selective depletion of the acetylcholine and vasoactive intestinal polypeptide of the guinea-pig myenteric plexus by differential mobilization of distinct transmitter pools. Exp. Brain Res. 72, 535-542. doi: 10.1007/BF00250599

Agoston, D. V., and Whittaker, V. P. (1989). Characterization, by size, density, osmotic fragility, and immunoaffinity, of acetylcholine- and vasoactive intestinal polypeptide-containing storage particles from myenteric neurones of the guinea-pig. J. Neurochem. 52, 1474-1480.

Allego, D., Gil, V., Aleu, J., Aulí, M., Clavé, P., and Jiménez, M. (2008). Purinergic and nitrergic junction potential in the human colon. Am. J. Physiol. Gastrointest. Liver Physiol. 295, G522-G533. doi: 10.1152/ajpgi.00510.2007

Anuras, S., Cooke, A. R., and Christensen, J. (1974). An inhibitory innervation at the gastroduodenal junction. J. Clin. Invest. 54, 529-535. doi: 10.1172/JCI107789

Atanasova, E. S., Vladimirova, I. A., and Shuba, M. F. (1972). [Nonadrenergic inhibitory postsynaptic potentials of stomach smooth muscle cells]. Neirofiziologiia 4, 216-222.

Bagkos, G., Koufopoulos, K., and Piperi, C. (2014). ATP synthesis revisited: new avenues for the management of mitochondrial diseases. Curr. Pharm. Des. 20, 4570-4579. doi: 10.2174/1381612819666131230124334

Belai, A., and Burnstock, G. (1994). Evidence for coexistence of ATP and nitric oxide in non-adrenergic, non-cholinergic (NANC) inhibitory neurones in the rat ileum, colon and anococcygeus muscle. Cell Tissue Res. 278, 197-200. doi: 10.1007/BF00305792

Bennett, M. R., Burnstock, G., and Holman, M. (1966). Transmission from intramural inhibitory nerves to the smooth muscle of the guinea-pig Taenia coli. J. Physiol. 182, 541-558. doi: 10.1113/jphysiol.1966.sp007836

Bennett, M. R., and Kearns, J. L. (2000). Statistics of transmitter release at nerve terminals. Prog. Neurobiol. 60, 545-606. doi: 10.1016/S0301-0082(99)00040-4

Burnstock, G. (1981). Review lecture. Neurotransmitters and trophic factors in the autonomic nervous system. J. Physiol. 313, 1-35. doi: 10.1113/jphysiol.1981.sp013648

Burnstock, G., Campbell, G., Satchell, D., and Smythe, A. (1970). Evidence that adenosine triphosphate or a related nucleotide is the transmitter substance released by non-adrenergic inhibitory nerves in the gut. Br. J. Pharmacol. 40, 668-688. doi: 10.1111/j.1476-5381.1970.tb10646.x

Chaudhury, A. (2014). Molecular handoffs in nitrergic neurotransmission. Front. Med. (Lausanne) 1:8. doi: 10.3389/fmed.2014.00008

Chaudhury, A. (2015a). A hypothesis for examining skeletal muscle biopsy-derived sarcolemmal $\mathrm{nNOS} \mu$ as surrogate for enteric nNOS $\alpha$ function. Front. Med. (Lausanne) 2:48. doi: 10.3389/fmed.2015.00048

Chaudhury, A. (2015b). Tail tale: nNOSdel1203-1434 predicts global defects in esophagogastrointestinal transit. Gastroenterology 149, 260-261. doi: 10.1053/j.gastro.2015.03.054

Chaudhury, A. (2016a). Furthering the debate on the role of interstitial cells of Cajal (ICCs) in enteric inhibitory neuromuscular neurotransmission. Am. J. Physiol. Cell Physiol. 311, C479-C481. doi: 10.1152/ajpcell.00067.2016

Chaudhury, A. (2016b). Response: “commentary: a hypothesis for examining skeletal muscle biopsy-derived sarcolemmal nNOS $\mu$ as surrogate for enteric nNOS $\alpha$ function." nNOS(skeletal muscle) may be evidentiary for enteric NOtransmission despite nNOS $\mu / \alpha$ differences. Front Med (Lausanne) 3:4. doi: $10.3389 /$ fmed.2016.00004

Chaudhury, A., He, X. D., and Goyal, R. K. (2009). Role of PSD95 in membrane association and catalytic activity of nNOSalpha in nitrergic varicosities in

\section{AUTHOR CONTRIBUTIONS}

AC conceptualized and drafted manuscript. VD important intellectual participation. WM important intellectual participation, overall supervision. All authors read and approved final version of manuscript.

mice gut. Am. J. Physiol. Gastrointest. Liver Physiol. 297, G806-G813. doi: 10.1152/ajpgi.00279.2009

Chaudhury, A., He, X. D., and Goyal, R. K. (2011). Myosin Va plays a key role in nitrergic neurotransmission by transporting $\mathrm{nNOS} \alpha$ to enteric varicosity membrane. Am. J. Physiol. Gastrointest. Liver Physiol. 301, G498-G507. doi: 10.1152/ajpgi.00164.2011

Chaudhury, A., He, X. D., and Goyal, R. K. (2012). Role of myosin Va in purinergic vesicular neurotransmission in the gut. Am. J. Physiol. Gastrointest. Liver Physiol. 302, G598-G607. doi: 10.1152/ajpgi.00330.2011

Collman, F., Buchanan, J., Phend, K. D., Micheva, K. D., Weinberg, R. J., and Smith, S. J. (2015). Mapping synapses by conjugate light-electron array tomography. J. Neurosci. 35, 5792-5807. doi: 10.1523/JNEUROSCI.4274-14.2015

Estévez-Herrera, J., Domínguez, N., Pardo, M. R., González-Santana, A., Westhead, E. W., Borges, R., et al. (2016). ATP: the crucial component of secretory vesicles. Proc. Natl. Acad. Sci. U.S.A. 113, E4098-E4106. doi: $10.1073 /$ pnas. 1600690113

González, A. A., Farré, R., and Clavé, P. (2004). Different responsiveness of excitatory and inhibitory enteric motor neurons in the human esophagus to electrical field stimulation and to nicotine. Am. J. Physiol. Gastrointest. Liver Physiol. 287, G299-G306. doi: 10.1152/ajpgi.00534.2003

Goyal, R. K., and Chaudhury, A. (2013). Structure activity relationship of synaptic and junctional neurotransmission. Auton. Neurosci. 176, 11-31. doi: 10.1016/j.autneu.2013.02.012

Goyal, R. K., Rattan, S., and Said, S. I. (1980). VIP as a possible neurotransmitter of non-cholinergic non-adrenergic inhibitory neurones. Nature 288, 378-380. doi: $10.1038 / 288378 \mathrm{a} 0$

Gwynne, R. M., and Bornstein, J. C. (2007). Mechanisms underlying nutrientinduced segmentation in isolated guinea pig small intestine. Am. J. Physiol. Gastrointest. Liver Physiol. 292, G1162-G1172. doi: 10.1152/ajpgi.00441.2006

Hirst, G. D., Bywater, R. A., Teramoto, N., and Edwards, F. R. (2004). An analysis of inhibitory junction potentials in the guinea-pig proximal colon. J. Physiol. 558(Pt 3), 841-855. doi: 10.1113/jphysiol.2004.065052

Jena, B. P. (2009). Functional organization of the porosome complex and associated structures facilitating cellular secretion. Physiology (Bethesda) 24, 367-376. doi: 10.1152/physiol.00021.2009

Jiménez, M., De Diego, M., Martínez-Cutillas, M., Mañé Reed, N., Gallego, D., Ojanguren, M. I., et al. (2015). Purinergic and nitrergic inhibitory neuromuscular transmission in ganglionic, transitional and aganglionic segments from Hirschsprung's disease patients. Neurogastroenterol. Motil. 27(S2):71Abs.

Katz, B. (1971). Quantal mechanism of neural transmitter release. Science 173, 123-126. doi: 10.1126/science.173.3992.123

Kopell, W. N., and Westhead, E. W. (1982). Osmotic pressures of solutions of ATP and catecholamines relating to storage in chromaffin granules. J. Biol. Chem. 257, 5707-5710.

Levine, D. N. (2007). Sherrington's "The integrative action of the nervous system": a centennial appraisal. J. Neurol. Sci. 253, 1-6. doi: 10.1016/j.jns.2006.12.002

Mackenzie, I., and Burnstock, G. (1980). Evidence against vasoactive intestinal polypeptide being the non-adrenergic, non-cholinergic inhibitory transmitter released from nerves supplying the smooth muscle of the guinea-pig taenia coli. Eur. J. Pharmacol. 67, 255-264. doi: 10.1016/0014-2999(80)90506-3

Sakamoto, S., Miyaji, T., Hiasa, M., Ichikawa, R., Uematsu, A., Iwatsuki, K., et al. (2014). Impairment of vesicular ATP release affects glucose metabolism and increases insulin sensitivity. Sci. Rep. 4:6689. doi: 10.1038/srep06689

Sawada, K., Echigo, N., Juge, N., Miyaji, T., Otsuka, M., Omote, H., et al. (2008). Identification of a vesicular nucleotide transporter. Proc. Natl. Acad. Sci. U.S.A. 105, 5683-5686. doi: 10.1073/pnas.0800141105

Smith, T. K., Bornstein, J. C., and Furness, J. B. (1990). Distension-evoked ascending and descending reflexes in the circular muscle of guinea-pig ileum: 
an intracellular study. J. Auton. Nerv. Syst. 29, 203-217. doi: 10.1016/01651838(90)90146-A

Südhof, T. C. (2013). Neurotransmitter release: the last millisecond in the life of a synaptic vesicle. Neuron 80, 675-690. doi: 10.1016/j.neuron.2013.10.022

Thorn, P., Zorec, R., Rettig, J., and Keating, D. J. (2016). Exocytosis in nonneuronal cells. J. Neurochem. 137, 849-859. doi: 10.1111/jnc.13602

Van Geldre, L. A., and Lefebvre, R. A. (2004). Interaction of NO and VIP in gastrointestinal smooth muscle relaxation. Curr. Pharm. Des. 10, 2483-2497. doi: 10.2174/1381612043383890

Whittaker, V. P. (1989). Vasoactive intestinal polypeptide (VIP) as a cholinergic co-transmitter: some recent results. Cell Biol. Int. Rep. 13, 1039-1051.
Conflict of Interest Statement: The authors declare that the research was conducted in the absence of any commercial or financial relationships that could be construed as a potential conflict of interest.

Copyright (c) 2016 Chaudhury, Dendi and Mirza. This is an open-access article distributed under the terms of the Creative Commons Attribution License (CC $B Y)$. The use, distribution or reproduction in other forums is permitted, provided the original author(s) or licensor are credited and that the original publication in this journal is cited, in accordance with accepted academic practice. No use, distribution or reproduction is permitted which does not comply with these terms. 